\title{
Identificación del perfil fitoquímico y efecto del estrés lumínico sobre la capacidad antioxidante del germinado de brócoli en un dispositivo germinador rotatorio tipo tambor
}

\author{
Identification of the phytochemical profile and effect of light stress on the antioxidant capacity of \\ broccoli sprouts in a drum type rotary germination
}

\begin{abstract}
Joel Hinojosa-Dávalos', Marco Antonio Cardona-López², Melesio Gutiérrez-Lomelí, Arturo Barrera-Rodrígueza Miguel Ángel Robles-García ${ }^{3 *}$

Departamento de Ciencias Básicas, Centro Universitario de la Ciénega. Universidad de Guadalajara. Av. Universisdad 1115, Ocotlán, Jalisco, México.

2 Laboratorio de Ciencias Médicas. Departamento de Ciencias Médicas y de la vida, Centro Universitario de la Ciénega. Universidad de Guadalajara. Av. Universidad 1115, Ocotlán, Jalisco, México.

3 Laboratorio de Alimentos. Departamento de Ciencias Médicas y de la vida, Centro Universitario de la Ciénega. Universidad de Guadalajara. Av. Universidad 1115, Ocotlán, Jalisco, México.
\end{abstract}

\section{RESUMEN}

La luz es uno de los factores ambientales más importantes que regulan el desarrollo de las plantas. El objetivo principal de este estudio fue determinar la influencia de diferentes condiciones de luz sobre la actividad antioxidante y el contenido de compuestos fenólicos en germinados de brócoli. Los germinados de brócoli fueron producidos en un germinador rotatorio con $10 \mathrm{~s}$ de aspersión de agua cada 90 min, 1 h de ventilación cada 3 h, 2 rpm, $99 \%$ HR y 3 condiciones de fotoperiodo: cada uno consistió en $11 \mathrm{~h}$ de luz blanca, además de $5 \mathrm{~h}$ luz roja, $5 \mathrm{~h}$ de luz azul o $5 \mathrm{~h}$ de luz verde, emitidos por focos Leds, dependiendo del tratamiento, y 8 $\mathrm{h}$ de oscuridad. La condición de luz roja obtuvo $0.71 \mathrm{~g} \mathrm{GS} / \mathrm{g}$ semilla diferencia significativa $(p<0.05)$. La luz azul obtuvo diferencia significativa $(p<0.05)$ en la inhibición de ambos radicales $A B T S$ y DPPH con una $E_{50}$ de $1.1232 \mathrm{mg} / \mathrm{mL}$ y $1.1577 \mathrm{mg} / \mathrm{mL}$ respectivamente. El mayor contenido de fenoles se obtuvo con la condición de luz roja de $10.17 \mathrm{mg}$ EAG/g y el contenido de flavonoides $1.86 \mathrm{mg} \mathrm{EQ} / \mathrm{g}$. Las diferentes condiciones de luz favorecen el incremento de compuestos bioactivos en germinados de brócoli en el dispositivo germinador rotatorio.

Palabras claves: Germinados, brócoli, estrés lumínico, capacidad antioxidante, germinador rotatorio.

\section{ABSTRACT}

Light is one of the most important environmental factors that regulate the development of plants. The main objective of this study was to determine the influence of different light conditions on the antioxidant activity and the phenolic compounds content in broccoli sprouts. The broccoli sprouts were produced in a rotary spreader with $10 \mathrm{~s}$ of water spraying every $90 \mathrm{~min}, 1 \mathrm{~h}$ of ventilation every $3 \mathrm{~h}, 2$ rpm, $99 \% \mathrm{RH}$ and 3 photoperiod conditions: each one consisted of $11 \mathrm{~h}$ of white light, in addition to $5 \mathrm{~h}$ of red light, $5 \mathrm{~h}$ of blue light or $5 \mathrm{~h}$ of green light, emitted by Leds, depending on the treatment, and $8 \mathrm{~h}$ of darkness. The red light condition

*Autor para correspondencia: Miguel Ángel Robles García

Correo electrónico: menen04@yahoo.com.mx

Recibido: 15 de noviembre de 2018

Aceptado: 19 de febrero de 2019 generated $0.71 \mathrm{~g} \mathrm{GS} / \mathrm{g}$ seed with a significant difference ( $p$ $<0.05$ ). The blue light obtained significant difference ( $p<$ 0.05 ) in the inhibition of both ABTS and DPPH radicals with an EC50 of $1.1232 \mathrm{mg} / \mathrm{mL}$ and $1.1577 \mathrm{mg} / \mathrm{mL}$ respectively. The highest phenols content was obtained with the red light condition of $10.17 \mathrm{mg} \mathrm{EA} / \mathrm{g}$ and the flavonoid content 1.86 $\mathrm{mg} E Q / \mathrm{g}$. The different light conditions favor the increase of bioactive compounds in broccoli sprouts in the rotating germination device.

Keywords: Sprout, broccoli, light stress, antioxidant capacity, drum rotary germinator.

\section{INTRODUCCIÓN}

Los germinados son de los pocos alimentos que se consumen cuando se encuentran en la etapa de desarrollo. Cobran importancia por el balance dietético y composición química (Sikin et al., 2013). En los primeros días de germinación las semillas de brócoli (Brassisca oleracea L.) alcanzan valores nutritivos de 10 a 100 veces más que las plantas en estado adulto (Fahey et al., 1997). Específicamente este germinado contiene compuestos fenólicos del tipo flavonoide, ácidos hidroxicinámicos, vitamina $A, C, E, K$, ácido fólico (B9), riboflavina (B2), hierro, calcio y potasio, indol-3-carbinol y sulforafano (Pérez-Balibrea et al., 2008; Moreno et al., 2010; Bjorkman et al., 2011; Manchali et al., 2012).

Los compuestos fenólicos son un grupo de moléculas pequeñas caracterizadas por sus estructuras que tienen al menos una unidad de fenol. En función de sus estructuras químicas, los compuestos fenólicos se pueden dividir en diferentes subgrupos, como ácidos fenólicos, flavonoides, taninos, cumarinas, lignanos, quinonas, estilbenos y curcuminoides (Gan et al., 2018). Se ha demostrado que algunos de estos compuestos juegan un papel importante en la salud humana, ya que tienen la capacidad para prevenir enfermedades degenerativas y puede disminuir el riesgo de ciertos tipos de cáncer como el cáncer de próstata, entre otros (Moreno et al., 2006; Gu et al., 2012), así como la diabetes (Ghawi et al., 2013). 
Por otro lado, se ha demostrado que una variedad de factores extrínsecos como: la temperatura, radiación, contaminación del aire, microorganismos, insectos y nutrientes, modifica en la planta la composición de metabolitos secundarios (Jahangir et al., 2009), alterando así la tolerancia al estrés (Mittler, 2002) y, por tanto, el valor nutricional (Verkerk et al., 2009; Rodríguez-Hernández et al., 2014). La luz es uno de los factores ambientales más importantes que regulan el crecimiento y desarrollo de las plantas tanto el desarrollo morfogénico, vegetativo y también el crecimiento de la plántula (Huché-Thélier et al., 2016). Estudios han demostrado que la calidad de la luz afecta el crecimiento y desarrollo de las plantas mediante la activación selectiva de diferentes fotorreceptores (Darko et al., 2014; Bian et al., 2015). Las propiedades únicas de los diodos emisores de luz (LED) permiten la manipulación conveniente de las características espectrales, la intensidad radiante o luminosa y los ajustes temporales de la luz producida (D'Souza et al., 2015). Se han utilizado LED de ancho de banda estrecho para analizar los cambios en los niveles de carotenoides y glucosinolatos (GL) en las coles maduras (Lefsrud et al., 2008) y en los niveles de nutrientes de germinados de brócoli con luz azul (Kopsell y Sams, 2013; Kopsell et al., 2014). Por otro lado, la producción de germinados implica el uso de tambores rotatorios, bandejas, estacionarios verticales o jarras de vidrio. Los tambores rotatorios utilizados industrialmente están diseñados para permitir la irrigación frecuente, el drenado sin restricciones, el tamaño de la carga de semillas, la velocidad y el tiempo de rotación (Fu et al., 2001; Tong-Jen et al., 2008). Por lo tanto, el objetivo de este estudio fue identificar el perfil fitoquímico del germinado de brócoli por estrés lumínico en un dispositivo germinador rotatorio tipo tambor.

\section{MATERIALES Y MÉTODOS Material vegetal}

Las semillas certificadas de Brócoli (Brassica oleracea L. var italica) fueron proporcionadas por una empresa de germinados de la región. Los germinados de brócoli fueron producidos en un dispositivo germinador rotatorio (registro de patente en trámite, No. de folio MX/E/2018/054797) bajo las condiciones de germinación de $10 \mathrm{~s}$ de aspersión de agua cada 90 min, 1 h de ventilación cada 3 h, 2 rpm, $99 \%$ humedad relativa y 3 condiciones de fotoperiodo: a) $11 \mathrm{~h} \mathrm{luz}$ blanca $5.84 \mu \mathrm{mol} / \mathrm{m}^{-2} \mathrm{~s}^{-2} / 5 \mathrm{~h}$ luz roja $628 \mathrm{~nm} 1.20 \mu \mathrm{mol} / \mathrm{m}$ ${ }^{2} \mathrm{~s}^{-2} / 8 \mathrm{~h}$ oscuridad; b) $11 \mathrm{~h}$ luz blanca $5.84 \mu \mathrm{mol} / \mathrm{m}^{-2} \mathrm{~s}^{-2} / 5 \mathrm{~h}$ luz azul $455 \mathrm{~nm}$ y $3.93 \mu \mathrm{mol} / \mathrm{m}^{-2} \mathrm{~s}^{-2} / 8 \mathrm{~h}$ oscuridad; c) $11 \mathrm{~h}$ luz blanca $5.84 \mu \mathrm{mol} / \mathrm{m}^{-2} \mathrm{~s}^{-2} / 5 \mathrm{~h}$ luz verde $521 \mathrm{~nm}$ y $3.79 \mu \mathrm{mol} / \mathrm{m}$ ${ }^{2} \mathrm{~s}^{-2} / 8 \mathrm{~h}$ oscuridad emitidos por diodos Leds, y como control se utilizó $16 \mathrm{~h}$ luz blanca/8 h oscuridad. Al finalizar 6 días de germinación, los germinados se colocaron en un secador de convección elaborado especialmente para este estudio, a 45 ${ }^{\circ} \mathrm{C}$ por $10 \mathrm{~h}$. Posteriormente, los germinados deshidratados fueron colocados en bolsas de poliestireno oscuras para la protección de la luz, finalmente, las muestras se colocaron en refrigeración.

\section{Obtención de extractos}

Para obtener los extractos se basó en la metodología de Morales-Del Río et. al (2015) con algunas modificaciones. Se pesaron $3 \mathrm{~g}$ de muestra seca y pulverizada, se adicionaron $30 \mathrm{~mL}$ de metanol, después las muestras se sonicaron (Sonicador BRANSONIC, $1510 \mathrm{R}-\mathrm{DTH}$ ) a $5{ }^{\circ} \mathrm{C}$ durante $15 \mathrm{~min}$, se centrifugaron a $10,000 \mathrm{rpm}$ por $10 \mathrm{~min}$ a $4{ }^{\circ} \mathrm{C}$ (Centrífuga Eppendorf, AG 22331 Hamburg, No. 5811YL843952). Las muestras se filtraron (papel filtro Whatman No. 1, poro 0.7 $\mu \mathrm{m}$ ) recuperándose el filtrado y el sedimento fue sometido a un segundo tratamiento con $30 \mathrm{~mL}$ de metanol, repitiendo el procedimiento posterior a la adición del metanol. Los filtrados obtenidos de cada muestra se concentraron en un rotavapor (Heidolph, 4003 VAC Senso T) a $40{ }^{\circ} \mathrm{C}$. El extracto de cada muestra se pesó para obtener el rendimiento de extracto por gramo de muestra seca y posteriormente se diluyó en metanol a una concentración final de $0.04 \mathrm{~g} / \mathrm{mL}$. A partir de esta preparación se determinaron la capacidad antioxidante, cuantificación de fenoles, flavonoides totales y la identificación colorimétrica de fenoles, saponinas, alcaloides, taninos y cumarinas de acuerdo a la metodología de Hinojosa-Dávalos et al. (2013).

\section{Determinación de la actividad antioxidante} ABTS (ácido 2,2'-azino-bis-(3-etilbenzotiazolina)-6 sulfonato de amonio). La determinación de radical $A B T S^{\circ+}$ se realizó por la técnica Re et al. (1999) con algunas modificaciones, se tomaron $270 \mu \mathrm{L}$ de la solución del radical catiónico preparado y se adicionaron $20 \mu \mathrm{L}$ de muestra leyendo a una absorbancia de $734 \mathrm{~nm}$ después de $30 \mathrm{~min}$ de reposo. Se utilizó metanol como control. Los resultados se expresaron en porcentaje de inhibición, calculado como se indica a continuación.

$$
\% \text { de inhibición }=\frac{\text { absorbancia control }- \text { absorbancia muestra }}{\text { absorbancia control }} \times 100
$$

DPPH (1,1-difenil-2-picrilhidrazil). Por medio de la técnica espectrofotométrica se estudió la capacidad de inhibición utilizando el radical sintético DPPH de acuerdo a la técnica de Molyneux (2004) con algunas modificaciones. Se tomaron $200 \mu \mathrm{L}$ de radical con $20 \mu \mathrm{L}$ de muestra, se dejó reposar media hora y se midió la absorbancia a una longitud de onda de $515 \mathrm{~nm}$. Se utilizó metanol como control. Los resultados se reportaron como porcentaje de inhibición, empleando la siguiente fórmula.

$$
\% \text { de inhibición }=\frac{\text { absorbancia control }- \text { absorbancia muestra }}{\text { absorbancia control }} \times 100
$$

Determinación de fenoles totales. Para la determinación de fenoles totales, se utilizó el método de Folin Ciocalteu utilizado por Prior et al. (2005) y Pérez-Nájera et al. (2013) con algunas modificaciones. Se tomaron $10 \mu \mathrm{L}$ de extracto y se agregaron $25 \mu \mathrm{L}$ de solución de Folin $1 \mathrm{~N}$ (SigmaAldrich). Se dejaron reposar por 5 min. Posteriormente, se adicionaron $25 \mu \mathrm{L}$ de $\mathrm{Na}_{2} \mathrm{CO}_{3}$ (Sigma-Aldrich)al $20 \%$ y $140 \mu \mathrm{L}$ de agua destilada para llegar a un volumen final de $200 \mu \mathrm{L}$. 
Se dejaron reposar 30 min y se determinó la absorbancia a $760 \mathrm{~nm}$. Se realizó una curva con el estándar ácido gálico en metanol. Los resultados se expresaron como miligramos de equivalentes de ácido gálico por gramo de muestra seca (mg EAG/gms). Todas las mediciones se realizaron por triplicado.

Determinación de flavonoides. La cuantificación de flavonoides se realizó mediante el método colorimétrico descrito por Maksimović et al. (2005) utilizando quercetina como control. Se tomaron alícuotas de $80 \mu \mathrm{L}$ de extracto y fueron adicionadas a $80 \mu \mathrm{L}$ de una solución etanólica de tricloruro de aluminio (20 g/L) (Sigma-Aldrich). Se agitaron durante 30 s y posteriormente se cubrieron con papel parafilm ${ }^{\circledR}$ dejándolo reposar durante $1 \mathrm{~h}$ en oscuridad a temperatura de 25 'C. Transcurrido este tiempo se volvió a agitar durante $30 \mathrm{~s}$ y se determinó la absorbancia a $415 \mathrm{~nm}$. Se realizó una curva de calibración con el estándar quercetina en metanol. Los resultados se expresan como miligramos de equivalentes de quercetina por gramo de muestra seca (mg EQ/gms).

\section{Identificación de fenoles mediante cromatografía en capa fina (TLC)}

La identificación de fenoles se realizó utilizando la técnica de García-Peña et al. (2009). Los extractos metanólicos de los germinados se colocaron en placas de TLC (7 x $4 \mathrm{~cm})$ (MACHEREY-NAGEL, DC-Fertigfolien ALUGRAM, 907189) para su identificación. A través de un capilar, se colocaron de 3 a 4 gotas de cada extracto correspondiente a una placa de TLC. Posteriormente, se dejó secar la placa con las muestras. Se colocó la fase móvil (éter de petróleo:acetato de etilo:ácido fórmico, 40:60:1) en la cámara cromatográfica con mínimo de 30 min para que impregnen los gases. Se sacaron las placas cuando el solvente eluyó a $1 \mathrm{~cm}$ de distancia del extremo final de dicha placa. Se dejó secar durante $5 \mathrm{~min}$, en seguida se llevó bajo luz UV (UVP, UVLS-26 El series UV Lamp, P/N 950279-0), a la longitud de onda de $254 \mathrm{~nm}$ para observar el número de compuestos fluorescentes. Posteriormente, se esparció a toda la placa con un atomizador el agente revelador para fenoles (o solución reveladora, $\mathrm{FeCl}_{3}$ al $10 \%$ ). Se dejó reposar 5 min y se colocó en una parrilla eléctrica (THERMO Scientific, SP131015) a $110{ }^{\circ} \mathrm{C}$ por un lapso de $10 \mathrm{~min}$. A continuación, se colocó la placa nuevamente bajo luz UV a la misma longitud de onda antes mencionada. Finalmente, se obtuvo el factor de retención ( $F R=$ distancia muestra / distancia solvente) de cada compuesto encontrado, se utilizó ácido gálico como control positivo.

\section{Identificación de flavonoides mediante cromatografía en capa fina (TLC)}

La identificación de flavonoides se realizó siguiendo el protocolo de García-Peña et al. (2009). Las placas de TLC, tuvieron las mismas características y el mismo procedimiento que para la identificación de fenoles mediante cromatografía en capa fina. La fase móvil fue acetato de etilo:ácido fórmico:ácido acético glacial:agua (100:11:11:27). Las placas de TLC fueron observadas con luz UV a una longitud de onda de $254 \mathrm{~nm}$. Se utilizó quercetina como control positivo. Por último, se obtuvo el FR de cada compuesto encontrado.

\section{Identificación de saponinas}

La identificación de saponinas se realizó por la técnica establecida por Galindo et al. (1989) y Sánchez-García et al. (2010). Se diluyó el extracto 1:9 tomando $1 \mathrm{~mL}$ del extracto metanólico colocándolo en un tubo de ensaye de vidrio con tapa $(13 \times 100 \mathrm{~mm})$. Se agregaron $9 \mathrm{~mL}$ de agua destilada, se homogenizó lo suficiente sin formar espuma y se filtró la disolución (papel filtro Whatman No.1). De esta mezcla se tomó $1 \mathrm{~mL}$ y se pasó a un tubo con tapa $(13$ x $100 \mathrm{~mm})$. Posteriormente, se agitó vigorosamente durante 30 s, se dejó reposar $15 \mathrm{~min}$. Transcurrido el tiempo establecido se midió la altura de la espuma formada en cada muestra.

\section{Prueba rápida para alcaloides}

Se utilizó $1 \mathrm{~mL}$ de extracto matanólico colocándolo en un crisol de porcelana, con la ayuda de una pipeta Pasteur se agregaron 4 gotas de hidróxido de amonio líquido (Sigma-Aldrich). Dicha solución se colocó bajo campana de extracción con el propósito de eliminar solventes. A este último residuo se le adicionaron tres gotas de ácido acético glacial (Sigma-Aldrich) y una gota de agua bidestilada, concentrando la solución nuevamente bajo campana de extracción. Por último, con la ayuda de una pipeta Pasteur se colocaron 4 gotas de la solución formada en un trozo de papel filtro (Whatman No.1) de $4 \times 4 \mathrm{~cm}$ y se agregaron 4 gotas del reactivo de Dragendorff. Se observó la coloración del papel filtro después de 24 horas. Como control positivo se utilizó cafiaspirina (la cual contiene el alcaloide cafeína). Un cambio de naranja a rojo o rosado sugiere la presencia de alcaloides (Galindo et al., 1989).

\section{Prueba rápida para taninos}

Esta prueba rápida se realizó bajo el protocolo propuesto por García-Peña et al. (2009) y Sánchez-García et al. (2010). Se colocaron aproximadamente $0.7 \mathrm{~g}$ de muestra seca en un matraz de afore. Se le añadieron $200 \mathrm{~mL}$ de ferrocianuro de potasio $\left(\mathrm{K}_{4} \mathrm{Fe}(\mathrm{CN})_{6}\right)$ para quedar finalmente a una concentración de $0.004 \mathrm{M}$, en ausencia de luz y sometidos a 100 rpm por 15 min (Shaker. Maxq2000, Barnstead/Lab-Line, SHKA2000). Transcurrido el tiempo señalado se adicionaron $20 \mathrm{~mL}$ de cloruro férrico $\left(\mathrm{FeCl}_{3}\right)$ al $0.008 \mathrm{M}$. Se considera un resultado positivos con cambio de coloración a) verde oscuro $=$ taninos condensados, b) azul = taninos hidrolizables.

\section{Prueba rápida para cumarinas}

Para determinar cumarinas se utilizó la técnica propuesta por Galindo et al. (1989), García-Peña et al. (2009) y Sánchez-García et al. (2010), con algunas modificaciones. A partir del extracto metanólico, se realizó una dilución siguiendo la proporción 1:9 de extracto:agua. Se colocaron de dicha dilución $2 \mathrm{~mL}$ en un tubo de vidrio con tapa, colocando una tira de papel filtro dentro del tubo previamente empapado de una solución alcalina de $\mathrm{NaOH}(0.06 \mathrm{~g} / \mathrm{mL})$. Sin tocar el 
extracto dentro del tubo, se tapó y se llevó a calentar en un mechero Bunsen hasta desprendimientos de vapores. Se tuvo el cuidado necesario para que el papel filtro no se quemara, ni se empapara de la solución alcalina al agitarse, evitando que sufriera proyección, ya que las cumarinas presentes en los extractos se acumulan en el papel filtro. Después, se llevó el papel filtro bajo lámpara UV (UVP, UVLS-26 El series UV Lamp, P/N 95-0279-0), observando la posible presencia de cumarinas, si el papel filtro exhibe puntos fluorescentes, la muestra se considera positiva.

\section{Análisis estadísticos}

Todos los datos fueron sujetos a un análisis de varianza (ANOVA) empleando el programa Statgraphics Centurion XV (ver.15.02.06.), los datos que se muestran son valores medios (media \pm desviación estándar). Las medias fueron comparadas usando la prueba de la diferencia mínima significativa (LSD) con un nivel de confianza del $95 \%$.

\section{RESULTADOS Y DISCUSIÓN}

Las condiciones de germinación a las que fueron sometidos los germinados se mantuvieron estables en los 6 días de experimentación. La cubierta protectora al ser de acrílico permitió el paso de luz blanca exterior, 523/interior 401 luxes, luz roja exterior 93/interior 82 luxes, luz azul exterior $320 /$ interior 270 y luz verde exterior 307/interior 260 luxes, a pesar de las diferencias de la intensidad de luz en el exterior e interior del germinador rotatorio las longitudes de onda de cada color se mantuvieron estables. Debido a que la luz es uno de los principales factores que afecta el crecimiento y morfogénesis en las plantas los tratamientos con diferentes condiciones de luz han estado bien documentados (Kopsell y Sams, 2013; Olle y Viršile, 2013; Carvalho y Folta, 2014; Craver et al., 2017). Las diferentes condiciones de luz con las que los germinados fueron irradiados en el dispositivo germinador rotatorio, mostraron diferencia estadística significativa $(p<$ 0.05 ) en todas las condiciones de luz aplicadas.

En este estudio, en la relación al peso seco, todas las condiciones tuvieron mayores rendimientos que la luz blanca, la condición de luz roja fue el que obtuvo mayor rendimiento, como se observa en la Tabla 1. Esto puede ser a que la emisión de luz roja ( 600 a 700 nm) se considera la más eficiente para impulsar la fotosíntesis en función del rendimiento cuántico de las mediciones en la fotosíntesis instantánea de la hoja (McCree, 1972). Sin embargo, en un estudio que fue realizado por Paniagua-Pardo et al. (2015) en 30 semillas de brócoli en un dispositivo germinador estacionario, reportaron incrementos del $11 \%$ con luz roja, $4 \%$ con azul y $17 \%$ con luz verde para el germinado de brócoli en fresco, y para el peso seco el mejor tratamiento fue el que utilizó luz roja con rendimientos hasta del $6 \%$ con respecto a la luz blanca. Estas diferencias entre las investigaciones pudieron deberse al tipo de dispositivo germinador y a la densidad de semillas utilizadas. Lo anterior puede deberse a que en el germinador rotatorio no solo las condiciones de luz son controladas sino también la humedad y la irrigación.
Tabla 1. Rendimiento de germinado de brócoli bajo las diferentes condiciones de luz en el germinador rotatorio.

Table 1. Broccoli sprout performance under different light conditions in the drum rotary germinator.

\begin{tabular}{lccccc}
\hline $\begin{array}{l}\text { Condiciones } \\
\text { de luz }\end{array}$ & $\begin{array}{c}\text { Semilla } \\
(\mathbf{g})\end{array}$ & GH (g) & GS (g) & $\begin{array}{c}\mathbf{g ~ G H / g} \\
\text { Semilla }\end{array}$ & $\begin{array}{c}\mathbf{g ~ G S / g} \\
\text { Semilla }\end{array}$ \\
\hline Blanca & 70 & $421 \pm 1^{\mathrm{b}}$ & $13 \pm 1.15^{\mathrm{d}}$ & $6 \pm 0.01^{\mathrm{b}}$ & $0.18 \pm 0.02^{\mathrm{d}}$ \\
\hline Roja & 70 & $385 \pm 1.15^{\mathrm{d}}$ & $50 \pm 1.52^{\mathrm{a}}$ & $5.5 \pm 0.01^{\mathrm{d}}$ & $0.71 \pm 0.02^{\mathrm{a}}$ \\
\hline Azul & 70 & $450 \pm 0.57^{\mathrm{a}}$ & $30 \pm 2^{\mathrm{b}}$ & $6.42 \pm 0.08^{\mathrm{a}}$ & $0.44 \pm 0.03^{\mathrm{b}}$ \\
\hline Verde & 70 & $416 \pm 1^{\mathrm{c}}$ & $22 \pm 1.5^{\mathrm{c}}$ & $5.94 \pm 0.01^{\mathrm{c}}$ & $0.33 \pm 0.02^{\mathrm{c}}$ \\
\hline
\end{tabular}

Abreviaciones: GH germinado húmedo; GS germinado seco

\pm Los valores representan las medias y las desviaciones estándar de tres repeticiones.

* Letras superíndice diferentes por columna indican diferencia significativa $(p<0.05)$.

Por otro lado, Kopsell et al. (2014), en microgreen (plántulas cosechadas de 7-14 días después de germinar) de brócoli germinados en charolas he iluminados con luz fluorescente/incandescente por un fotoperiodo de $16 \mathrm{~h}$ con $5 \%$ $\mathrm{azul} / 85 \% \mathrm{roja} / 10 \%$ verde por 21 días obtienen rendimientos $11.26 \mathrm{~g}$ por gramo de semilla, por el contrario a los resultados obtenidos en nuestro estudio con las condiciones de luz aplicadas se obtuvieron entre 5.5-6.42 g de germinado por gramos de semilla de brócoli. Cabe mencionar que la diferencia puede ser por el tiempo de crecimiento de las plantas considerando que el germinado dura 6 días en proceso de germinación y el microgreen más de 9 días de crecimiento o hasta la emergencia de las hojas verdaderas. Por otro lado, los rendimientos de extractos metanólicos no mostraron diferencia significativa $(p>0.05)$ entre los tratamientos con respecto a la condición de luz blanca, como lo muestra la Tabla 2.

Los extractos de plantas se obtienen para concentrar los metabolitos y/o compuestos fitoquímicos presentes en los vegetales, entre estos se encuentran alcaloides, saponinas, compuestos fenólicos, etc. los cuales pueden poseer actividad antialergénica, antiinflamatoria, antihipertensiva,

Tabla 2. Rendimiento de extractos metanólicos de los germinados de brócoli bajo las diferentes condiciones de luz.

Table 2. Performance of methanolic extracts of the broccoli sprouts under the different light conditions.

\begin{tabular}{lcccc}
\hline $\begin{array}{l}\text { Condiciones } \\
\text { de luz }\end{array}$ & $\begin{array}{c}\text { muestra } \\
\text { seca }(\mathbf{g})\end{array}$ & Extracto $(\mathbf{g})$ & $\mathbf{g ~ E / g ~ m s}$ & $\%(\mathbf{p} / \mathbf{p})$ \\
\hline Blanca & 3 & $0.51 \pm 0.10^{\mathrm{a}}$ & $0.12 \pm 0.02^{\mathrm{a}}$ & $12 \pm 1.7^{\mathrm{a}}$ \\
\hline Rojo & 3 & $0.44 \pm 0.03^{\mathrm{a}, \mathrm{b}}$ & $0.12 \pm 0.01^{\mathrm{a}}$ & $12.1 \pm 0.77^{\mathrm{a}}$ \\
\hline Azul & 3 & $0.40 \pm 0.03^{\mathrm{a}, \mathrm{b}}$ & $0.11 \pm 0.00^{\mathrm{a}}$ & $11.05 \pm 0.40^{\mathrm{a}}$ \\
\hline Verde & 3 & $0.31 \pm 0.09^{\mathrm{b}}$ & $0.10 \pm 0.02^{\mathrm{a}}$ & $10 \pm 2.2^{\mathrm{a}}$ \\
\hline
\end{tabular}

Abreviaciones: g E gramo extracto; ms muestra seca

\pm Los valores representan las medias y las desviaciones estándar de tres repeticiones.

* Letras superíndice diferentes por columna indican diferencia significativa $(p<0.05)$. 
antiviral, antifúngica, así como también la capacidad de inhibir radicales libres (Ramírez-Rojo et al., 2018). De acuerdo con los resultados obtenidos en las pruebas cualitativas, se observó la presencia de los metabolitos secundarios en los germinados de brócoli bajo todas las condiciones de luz, sin la modificación de éstos, por la aplicación de las diferentes condiciones de luz con respecto a la luz blanca (Tabla 3). Resultados similares han sido obtenidos por Chauhan et al. (2016) en repollo morado, reportan la presencia de flavonoides, taninos y la ausencia de saponinas y cumarinas en extractos metanólicos y Guriya et al. (2015) en repollo verde, reportaron la presencia de alcaloides, flavonoides, taninos, fenoles y ausencia de saponinas. Caso contrario en un estudio realizado por Kusmiati y Agustini (2017) en hojas de brócoli secas y maceradas con $n$-hexano por $48 \mathrm{~h}$ obtuvieron la presencia de saponinas y ausencia de fenoles, taninos y alcaloides. Así mismo, Dhiman et al. (2015) en extractos metanólicos de hojas de brócoli reportan la ausencia de alcaloides, fenoles y taninos y la presencia de saponinas. Se podría sugerir que estas diferencias se deben al estado fenológico de las plantas y los germinados, esto debido a que a medida que progresa la maduración de la planta se sintetizan o activan enzimas en etapas particulares del desarrollo de la planta que participan en la síntesis de metabolitos secundarios (Chen et al., 2012).

Los extractos metanólicos de los germinados de brócoli fueron evaluados por cromatografía en capa fina (TLC por sus siglas en inglés) para la determinación de fenoles y flavonoides como se muestra en la Tabla 4, con la presencia de compuestos solubles en referencia al disolvente empleado y de su factor de retención (FR). Sin embargo, puede existir la posibilidad de compuestos similares, como los isómeros o muy parecidos en su estructura y por tanto tener un FR muy similar, por lo tanto, una TLC solo muestra un perfil probable sobre el número de compuestos, pero no da la seguridad absoluta del total de compuestos existentes en los germinados. Los resultados obtenidos mostraron que los extractos metánolicos de germinados de brócoli expuestos a diferentes condiciones de luz correspondían a los estándares de ácido gálico compuesto No. 6 y quercetina compuesto No. 3 , con un FR de 0.4 y 0.7 respectivamente.

Es ampliamente conocido que los efectos de la intensidad y calidad de la luz tiene efectos positivos en la acumulación de fitoquímicos en plantas (Li y Kubota, 2009; Fu et al., 2012). La irradiación durante el cultivo de germinados aumenta el contenido fenólico total al mejorar la fotosíntesis y la ruta de la malonil-CoA, que se asocia con la síntesis de

Tabla 3. Interpretación colorimétrica de metabolitos secundarios de los extractos metanólicos de los germinados de brócoli.

Table 3. Colorimetric interpretation of secondary metabolites of the broccoli sprouts methanolic extracts.

\begin{tabular}{lcccccc}
\hline $\begin{array}{l}\text { Condiciones } \\
\text { de Luz }\end{array}$ & Fenoles & Flavonoides & Saponinas & Alcaloides & Taninos & Cumarinas \\
\hline Blanca & + & + & - & - & + & - \\
Roja & + & + & - & - & + & - \\
Azul & + & + & - & - & + & - \\
Verde & + & + & - & - & + & - \\
\hline
\end{tabular}

Presencia (+), Ausencia (-)

Tabla 4. Valores de retención FR (factor de retención) observado en fenoles y flavonoides de los extractos metanólicos de germinado de brócoli por cromatografía en capa fina (TLC).

Table 4. RF retention values (retention factor) observed in phenols and flavonoids of methanol extracts of broccoli sprout by thin layer chromatography (TLC).

\begin{tabular}{|c|c|c|c|c|c|c|c|c|c|c|}
\hline \multirow{3}{*}{$\begin{array}{l}\text { No. de } \\
\text { Compuesto } \\
\text { (s) }\end{array}$} & \multicolumn{5}{|c|}{ Fenoles } & \multicolumn{5}{|c|}{ Flavonoides } \\
\hline & \multicolumn{5}{|c|}{$254 \mathrm{~nm}$} & \multicolumn{5}{|c|}{$254 \mathrm{~nm}$} \\
\hline & AG & C & $\mathbf{R}$ & A & v & $\mathbf{Q}$ & C & $\mathbf{R}$ & A & v \\
\hline 1 & - & 0.9 & 0.9 & 0.9 & 0.9 & - & - & - & - & - \\
\hline 2 & - & - & 0.8 & 0.8 & 0.8 & - & - & - & - & - \\
\hline 3 & - & 0.7 & 0.7 & 0.7 & 0.7 & 0.7 & 0.7 & 0.7 & 0.7 & 0.7 \\
\hline 4 & - & 0.6 & 0.6 & 0.6 & 0.6 & - & - & - & - & - \\
\hline 5 & - & - & - & - & - & - & 0.5 & 0.5 & 0.5 & 0.5 \\
\hline 6 & 0.4 & - & 0.4 & 0.4 & 0.4 & - & 0.4 & 0.4 & 0.4 & 0.4 \\
\hline 7 & - & - & - & - & - & - & 0.3 & 0.3 & 0.3 & 0.3 \\
\hline 8 & - & - & - & - & - & - & 0.2 & 0.2 & 0.2 & 0.2 \\
\hline
\end{tabular}

Abreviaciones: (AG) Ácido gálico; (Q) quercetina; (C) luz blanca; (R) luz roja; (A) luz azul; (V) luz verde. 
compuestos fenólicos (Kim et al., 2005; Pérez-Balibrea et al., 2008). Así mismo, Long y Jenkins (1998) observaron que la luz azul induce a las enzimas involucradas en el metabolismo fenólico, como la fenilalanina amoníaco-liasa y la chalcona sintasa. Así mismo, Qian et al. (2016) en su estudio con luz azul en el germinado de Kale chino indujo la acumulación de antiocianidinas de fenoles y flavonoides. En otro estudio, Kwack et al. (2015) examinaron el efecto de la intensidad y calidad luz LED en germinados de brócoli y observaron que el contenido de fenoles fue mayor en las condiciones de luz roja y azul.

Por otra parte, la actividad antioxidante de los extractos de brócoli contra los radicales libres de ABTS y DPPH fue por arriba del $85 \%$ en todas las condiciones de luz. La capacidad de inhibición de los radicales ABTS y DPPH por los extractos mostraron diferencia significativa entre ellos ( $p$ $<0.05$ ), con el radical ABTS se obtuvieron mayores porcentajes de inhibición, esto podría ser debido a que el ABTS no interfiere con la absorción de los compuestos que absorben a longitudes de onda del espectro visible y por su afinidad por compuestos hidrofílicos e hidrofóbicos presentes en los extractos (Solanilla et al., 2011) (Figura 1). Por su parte la capacidad antioxidante de los extractos juega un papel importante, se puede decir que esta propiedad va a depender de la estructura química de los compuestos y de la presencia de grupos hidroxilo presentes en la molécula. Los grupos hidroxilo, al estar unidos a un anillo bencénico, presentan la posibilidad de que el radical libre interaccione con los electrones del anillo, lo que les confiere características especiales respecto al resto de alcoholes (Perez-Perez et al., 2018). Sin embargo, la condición de luz azul es la que obtuvo mayor efecto en la inhibición de los radicales ABTS y DPPH como

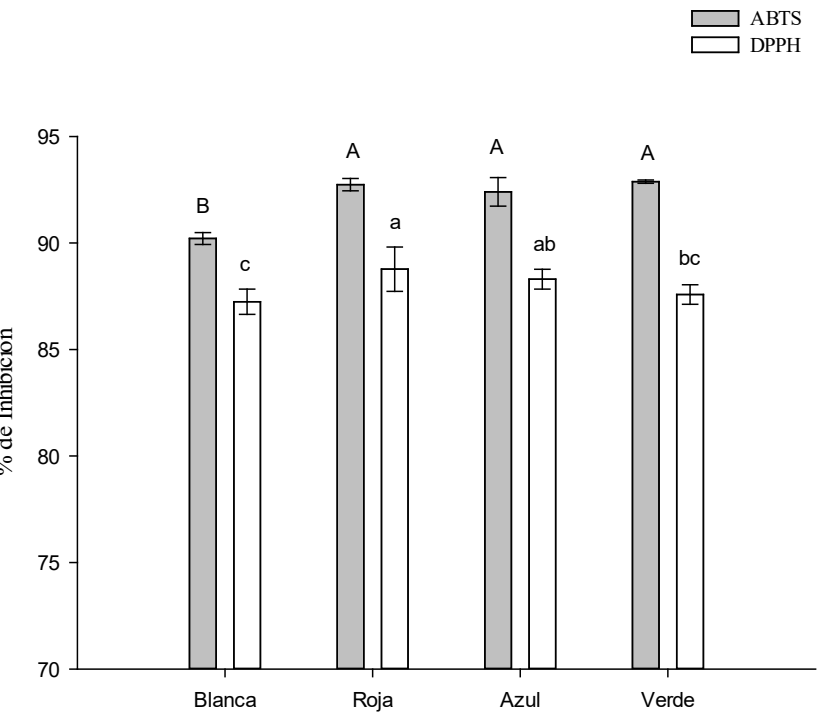

Figura 1. Porcentaje de inhibición de los radicales libres ABTS y DPPH de los extractos metanólicos de germinados de brócoli. Letras diferentes mayúsculas (ABTS) y minúsculas (DPPH) indican diferencia significativa $(p<0.05)$ entre los tratamientos.

Figure 1. Percentage of inhibition of the ABTS and DPPH free radicals of broccoli sprouts methanol extracts. Different uppercase letters (ABTS) and lowercase letters (DPPH) indicate significant difference $(p<0.05)$ between treatments. lo muestra la $\mathrm{EC}_{50}$ en muestra seca (Tabla 5). Los porcentajes de inhibición del radical ABTS por los extractos metanólicos de los germinados de brócoli bajo las diferentes condiciones de luz se pueden observar en la figura 1. De acuerdo con los resultados obtenidos, el porcentaje de inhibición fluctúa entre 90 y $92 \%$. En las 3 condiciones de luz se presentaron diferencias significativas $(p<0.05)$ con respecto a la luz blan$c a$, siendo esta misma la de menor porcentaje de inhibición, en cambio las luces roja, azul y verde presentan el mayor porcentaje de inhibición con un $2 \%$ más que la luz blanca.

Tabla 5. $\mathrm{EC}_{50}$ de los extractos metanólicos de los germinados de brócoli contra los radicales libres de ABTS Y DPPH.

Table 5. $\mathrm{EC}_{50}$ of the methanolic extracts of broccoli sprouts against the $A B T S$ and DPPH free radicals.

\begin{tabular}{lcc}
\hline Condición de luz & $\mathrm{EC}_{\mathbf{5 0}}$ ABTS $\mathbf{m g} / \mathbf{m L}$ & EC $_{50}$ DPPH $\mathbf{~ g} / \mathbf{m L}$ \\
\hline Control & 1.5068 & 2.0789 \\
Rojo & 1.2506 & 1.5271 \\
Azul & 1.1232 & 1.1577 \\
Verde & 1.5254 & 1.9928 \\
\hline
\end{tabular}

El porcentaje de inhibición del radical DPPH fluctúa entre los 86 y $88 \%$ como lo muestra la Figura 1. Resultados diferentes obtenidos con el radical ABTS. Sin embargo, la condición luz roja y azul están por arriba del porcentaje de inhibición de la luz blanca excepto la luz verde que no mostró diferencia significativa con respecto a la luz blanca.

En un estudio realizado por Cho et al. (2008) obtuvieron una reducción del radical libre DPPH del $93.5 \%$ con luz blanca en germinados de brócoli a los 8 días de geminación, en comparación por los resultados obtenidos en nuestro estudio de $87.2 \%$, haciendo así una diferencia de $6.29 \%$ menos de inhibición del radical DPPH con germinados de brócoli con luz blanca. Resultados similares a nuestro estudio se obtuvieron por Chaudhary et al. (2016) con germinados de coliflor bajo los fotoperiodos de $16 \mathrm{~h}$ luz blanca y $8 \mathrm{~h}$ oscuridad a $24{ }^{\circ} \mathrm{C}$ donde obtuvieron $87.70 \%$ de inhibición con el radical DPPH a los 5 días y $80.90 \%$ a los 7 días de germinación y una $I C_{50}$ de $1.51 \mathrm{mg} / \mathrm{mL}$. Vale et al. (2014) obtuvieron una IC ${ }_{50} 0.217$ $\mathrm{mg} / \mathrm{mL}$ a los 7 días de germinación de semillas brócoli en un gabinete de germinación con ciclos de fotoperiodos de 16 $\mathrm{h}$ luz blanca y $8 \mathrm{~h}$ de oscuridad. En nuestro estudio bajo la condición de luz verde se obtuvo de $1.99 \mathrm{mg} / \mathrm{mL}$ en el germinado de brócoli con 5 días de germinación. Por otro lado, las diferencias encontradas pueden deberse al mecanismo de reacción de los radicales con los compuestos de los extractos estudiados. El radical DPPH puede reaccionar por la sucesión de un átomo de hidrógeno por el agente antioxidante en una reacción de pseudo primer orden en la que puede ocurrir una dimerización de los productos formados o a reacciones de los productos de la misma reacción (Guija-Poma et al., 2015). Por otro lado, el radical ABTS puede reaccionar con especies donantes de hidrógeno o electrones y tanto la estructura química, la concentración del compuesto, la posición de los grupos hidroxilo y el tiempo, determinarán el orden de la 
reacción (Tian y Schaich, 2013). Las diferencias encontradas con respecto a la cantidad de fenoles por la técnica de FollinCiocalteu en cada condición aplicada se pueden observar en la Figura 2. De acuerdo con los resultados obtenidos las condiciones de luz roja fue la que obtuvo una mayor concentración de fenoles similar a la condición de luz blanca con diferencia significativa con respecto a la condición de luz verde. Similar al resultado obtenido para los flavonoides la condición de luz roja fue la que mayor concentración de flavonoides obtuvo, mostrando una diferencia significativa con respecto al resto de las condiciones de luz (Figura 2).

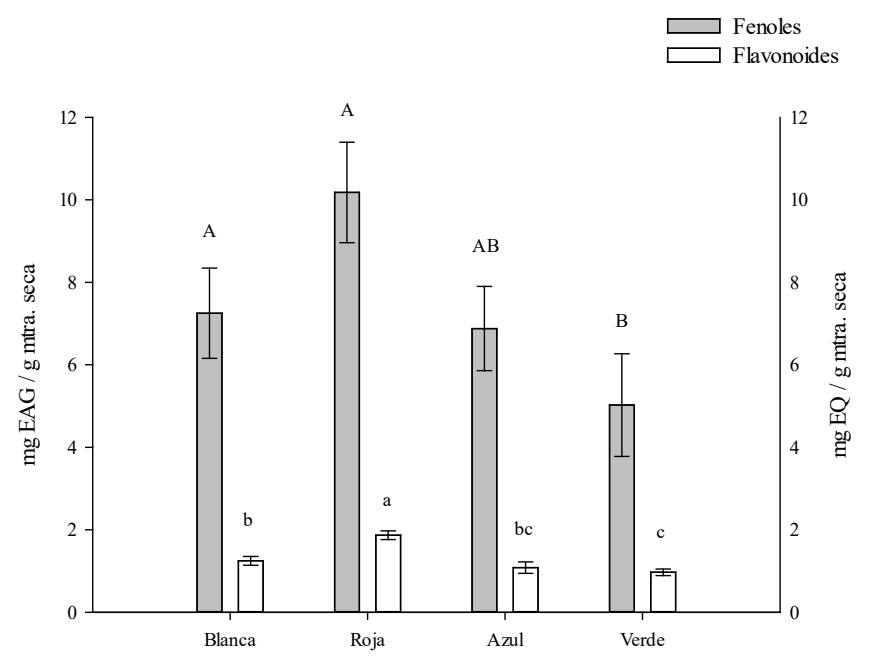

Figura 2. Fenoles ( $m g$ EAG/g mtra. seca) y Flavonoides ( $\mathrm{mg} \mathrm{EQ} / \mathrm{g}$ mtra. seca) totales in vitro de los extractos metanólicos de los germinados de brócoli. Letras diferentes mayúsculas (fenoles) y minúsculas (flavonoides) indican diferencia significativa $(p<0.05)$ entre los tratamientos.

Figure 2. In vitro total phenols (mg EAG/g dry sample) and flavonoids ( $\mathrm{mg}$ $\mathrm{EQ} / \mathrm{g}$ dry sample) of the methanolic extracts of broccoli sprouts. Different uppercase letters (phenols) and lowercase letters (flavonoids) indicate significant difference $(p<0.05)$ between treatments.

En un estudio realizado por Vale et al. (2014) utilizaron un germinador estacionario vertical y no obtuvieron conteo de fenoles totales a los 6 días de germinación en germinados de brócoli, sin embargo, después de 15 días de germinación obtuvieron 24 mg EQ de muestra seca. La extracción de dichos compuestos la realizaron con agua destilada a diferencia de nuestro estudio que la extracción de los compuestos se realizó con metanol, obteniendo $1.8 \mathrm{mg} E \mathrm{EQ}$ de muestra seca a los 6 días de germinación, por lo que las diferencias entre ambos estudios pueden deberse por el tipo de extracción utilizada. En un estudio diferentes, Pérez-Balibrea et al. (2008) obtuvieron $310.2 \mathrm{mg}$ por $100 \mathrm{~g}$ de muestra fresca de compuestos fenólicos en extractos metanólicos de germinados de brócoli cultivados por $16 \mathrm{~h}$ luz blanca/8 h oscuridad durante 7 días. Aunque los flavonoides son los polifenoles mayormente distribuidos en las plantas (Martínez-Valverde et al., 2000) no necesariamente quiere decir que un alto contenido de fenoles indica un alto contenido de flavonoides (Bedascarrasbure et al., 2004).

\section{CONCLUSIONES}

El dispositivo germinador rotatorio permite mantener las condiciones de humedad relativa, irrigación y luminosidad dentro de la cámara de germinación para el desarrollo óptimo de germinado de brócoli, así mismo, en la condición de luz roja se obtienen los mejores rendimientos de germinados, favorece el aumento de fenoles y flavonoides. El dispositivo germinador rotatorio puede ser potencialmente utilizado para que las semillas germinadas aumenten el contenido de compuestos fitoquímicos, y con ellos tener un valor agregado en la producción de alimentos con alto contenido de antioxidantes, convirtiéndolos en una mejor fuente de este tipo de compuestos bioactivos.

\section{AGRADECIMIENTOS}

A CONACYT por el apoyo del becario del Doctorado en Ciencias M. en C. Joel Hinojosa Dávalos C.V.U. 354415.

\section{REFERENCIAS}

Bedascarrasbure, E., Maldonado, L., Álvarez, A. y Rodríguez, E. 2004. Contenido de fenoles y flavonoides del propoleos Argentino. Acta Farmacéutica Bonaerense. 23: 369-372.

Bian, Z.H., Yang, Q.C. y Liu, W.K. 2015. Effects of light quality on the accumulation of phytochemicals in vegetables produced in controlled environments: a review. Journal of the Science of Food and Agriculture. 95: 869-877.

Bjorkman, M., Klingen, I., Birch, A.N., Bones, A.M., Bruce, T.J., Johansen, T.J., Meadow, R., Molmann, J., Seljasen, R., Smart, L.E. y Stewart, D. 2011. Phytochemicals of Brassicaceae in plant protection and human health--influences of climate, environment and agronomic practice. Phytochemistry. 72: 538-556.

Carvalho, S.D. y Folta, K.M. 2014. Sequential light programs shape kale (Brassica napus) sprout appearance and alter metabolic and nutrient content. Horticulture Research. 1: 8.

Chaudhary, A., Choudhary, S., Sharma, U., Vig, A. y Arora, S. 2016. In vitro Evaluation of Brassica sprouts for its Antioxidant and Antiproliferative Potential. Indian Journal of Pharmaceutical Sciences. 78: 615-623.

Chauhan, E.S., Tiwari, A. y Singh, A. 2016. Phytochemical screening of red cabbage (Brassica oleracea) powder and juice-A comparative study. Journal of Medicinal Plants Studies. 4: 196-199.

Chen, Y., Zhu, Z., Guo, Q., Zhang, L. y Zhang, X. 2012. Variation in concentrations of major bioactive compounds in Prunella vulgaris L. related to plant parts and phenological stages biology research. 45: 171-175.

Cho, J.-Y., Son, D.-M., Kim, J.-M., Seo, B.-S., Yang, S.-Y., Bae, J.-H. y Heo, B.-G. 2008. Effect of LED as light quality on the germination, growth and physiological activities of broccoli sprouts. Journal of Bio-Environment Control. 17: 117-123.

Craver, K.J., Gerovac, J., Lopez, R. y Kopsell, D. 2017. Light Intensity and Light Quality from Sole-source Light-emitting Diodes Impact Phytochemical Concentrations within Brassica Microgreens. Journal of the American Society for Horticultural Science. 142: 3-12.

D'souza, C., Yuk, H.-G., Khoo, G.H. y Zhou, W. 2015. Application of Light-Emitting Diodes in Food Production, Postharvest Preservation, and Microbiological Food Safety. 
Comprehensive Reviews in Food Science and Food Safety. 14: 719-740.

Darko, E., Heydarizadeh, P., Schoefs, B. y Sabzalian, M.R. 2014. Photosynthesis under artificial light: the shift in primary and secondary metabolism. Philosophical Transaction of the Royal Socyety B. 369: 20130243.

Dhiman, M.I., Prashar, M.Y., Kalia, M.K. y Gill, N.S. 2015. Therapeutic And Nutritional Value Of Brassica Oleracea L. Var. Italica (Broccoli): A Review. International Journal Of Universal Pharmacy And Bio Sciences. 4: 22-23.

Fahey, J.W., Zhang, Y. y Talalay, P. 1997. Broccoli sprouts: an exceptionally rich source of inducers of enzymes that protect against chemical carcinogens. Proceedings of the National Academy of Sciences of the United States of America. 94: 10367-10372.

Fu, T., Stewart, D., Reineke, K., Ulaszek, J., Schelsser, J. y Tortorelo, M. 2001. Use of spent irrigation water for microbiological analysis of alfalfa sprouts. Journal of Food Protection. 64: 802-806.

Fu, W., Li, P. y Wu, Y. 2012. Effects of different light intensities on chlorophyll fluorescence characteristics and yield in lettuce. Scientia Horticulturae. 135: 45-51.

Galindo, W.F., Rosales, M., Murgueitio, E. y Larrahondo, J. 1989. Sustancias antinutricionales en las hojas de Guamo, Nacedero y Matarratón. Livestock Research for Rural Development. 1: 36-47.

Gan, R.-Y., Chan, C.-L., Yang, Q.-Q., Li, H.-B., Zhang, D., Ge, Y.-Y., Gunaratne, A., Ge, J. y Corke, H. 2018. 9 - Bioactive compounds and beneficial functions of sprouted grains. In: Feng, H., Nemzer, B. y Devries, J.W. (eds.) Sprouted Grains. AACC International Press. 191-246.

García-Peña, C.M., Kim Bich, N., Bich Thu, N., Tillan Capo, J., Romero Díaz, J.A., López, O.D. y Fuste Moreno, V. 2009. Metabolitos secundarios en los extractos secos de Passiflora incarnata L., Matricaria recutita L. y Morinda citrifolia L. Revista Cubana de Plantas Medicinales. 14: 0-0.

Ghawi, S.K., Methven, L. y Niranjan, K. 2013. The potential to intensify sulforaphane formation in cooked broccoli (Brassica oleracea var. italica) using mustard seeds (Sinapis alba). Food Chemestry. 138: 1734-1741.

Gu, Y., Guo, Q., Zhang, L., Chen, Z., Han, Y. y Gu, Z. 2012. Physiological and biochemical metabolism of germinating broccoli seeds and sprouts. Journal of Agricultural of Food Chemestry. 60: 209-213.

Guija-Poma, E., Inocente-Camones M.A., Ponce-Pardo, J. y Zarzosa-Norabuena. 2015. Evaluación de la técnica 2,2-Difenil-I-Picrilhidrazilo (DPPH) para determinar la capacidad antioxidante. Horizonte Médico. 15: 56-60.

Guriya, R., Moon, A. y Talreja, K. 2015. Phytochemical profiling and characterization of bioactive compounds from Brassica oleracea. International Journal of Pharmacognosy and Phytochemical Research. 7: 825-831.

Hinojosa-Dávalos, J., Gutiérrez-Lomelí, M., Siller-López, F., Rodríguez-Sahagún, A., Morales-Del- Río, J.A., GuerreroMedina, P.J., Del-Toro-Sánchez, C.L. (2013). Phytochemycal screening and antiinflammatory capacity of leaves from Tithonia tubaeformis. Biotecnia. 15(2): 53-60.Huché-Thélier, L., Crespel, L., Gourrierec, J.L., Morel, P., Sakr, S. y Leduc, N. 2016. Light signaling and plant responses to blue and UV radiations-Perspectives for applications in horticulture. Environmental and Experimental Botany. 121:22-38.
Jahangir, M., Abdel-Farid, I.B., Kim, H.K., Choi, Y.H. y Verpoorte, R. 2009. Healthy and unhealthy plants: The effect of stress on the metabolism of Brassicaceae. Environmental and Experimental Botany. 67: 23-33.

Kim, E.H., Kim, S.H., Chung, J.I., Chi, H.Y., Kim, J.A. y Chung, I.M. 2005. Analysis of phenolic compounds and isoflavones in soybean seeds (Glycine max (L.) Merill) and sprouts grown under different conditions. European Food Research and Technology. 222: 201-208.

Kopsell, D.A. y Sams, C.E. 2013. Increases in shoot tissue pigments, glucosinolates, and mineral elements in sprouting broccoli after exposure to short-duration blue light from light emitting diodes. Journal of the American Society For Horticultural Science. 138: 31-37.

Kopsell, D.A., Sams, C.E., Barickman, T.C. y Morrow, R.C. 2014. Sprouting broccoli accumulate higher concentrations of nutritionally important metabolites under narrow-band light-emitting diode lighting. Journal of the American Society for Horticultural Science. 139: 469-477.

Kusmiati y Agustini, S.N.W. 2017. Potential Lutein Extract of Broccoli (Brassica oleracea L. var. italica) as Antiradical Abts (2,2 - Azinobis Acid, 3-Ethyl Benzothiazoline-6-Sulfonic Acid). 2017. 11.

Kwack, Y., Kim, K.K., Hwang, H. y Chun, C. 2015. Growth and quality of sprouts of six vegetables cultivated under different light intensity and quality. Horticulture, Environment, and Biotechnology. 56: 437-443.

Lefsrud, M.G., Kopsell, D.A. y Sams, C.E. 2008. Irradiance from distinct wavelength light-emitting diodes affect secondary metabolites in kale. HortScience. 43: 2243-2244.

Li, Q. y Kubota, C. 2009. Effects of supplemental light quality on growth and phytochemicals of baby leaf lettuce. Environmental and Experimental Botany. 67: 59-64.

Long, J.C. y Jenkins, G.I. 1998. Involvement of plasma membrane redox activity and calcium homeostasis in the UV-B and UV-A/blue light induction of gene expression in Arabidopsis. Plant Cell. 10: 2077-2086.

Maksimović, Z., Malenčić, Đ. y Kovačević, N. 2005. Polyphenol contents and antioxidant activity of Maydis stigma extracts. Bioresource Technology. 96: 873-877.

Manchali, S., Chidambara Murthy, K.N. y Patil, B.S. 2012. Crucial facts about health benefits of popular cruciferous vegetables. Journal of Functional Foods. 4: 94-106.

Martínez- Valverde, I., Periago, M.J. y Ros, G. 2000. Significado nutricional de los compuestos fenólicos de la dieta. Archivos Latinoamericanos de Nutrición. 50: 5-18.

McCree. 1972. The action spectrum, absorptance and quantum yield of photosynthesis in crop plants. Agricultural Meteorology. 9: 191-216.

Mittler, R. 2002. Oxidative stress, antioxidants and stress tolerance. Trends in Plant Science. 7: 405-410.

Molyneux, P. 2004. The use of the stable free radical diphenylpicrylhydrazyl (DPPH) for estimating antioxidant activity. Songklanakarin Journal Science and Technology. 26: 211-219.

Morales-Del-Rio, J.A., Gutiérrez-Lomelí, M., Robles-García, M.A., Aguilar, J.A., Lugo-Cervantes, E., Guerrero-Medina, P.J., RuizCruz, S., Cinco-Moroyoqui, F.J., Wong-Corral, F.J., Del-ToroSánchez, C.L. 2015. Anti-inflammatory activity and changes in antioxidant properties of leaf and stem extracts from Vitex mollis Kunth during in vitro digestion. Evidence-Based 
Complementary and Alternative Medicine (eCAM). 2015. Doi: http://dx.doi.org/10.1155/2015/349235.

Moreno, D.A., Carvajal, M., Lopez-Berenguer, C. y GarciaViguera, C. 2006. Chemical and biological characterisation of nutraceutical compounds of broccoli. Journal of Pharmceutical Biomedical Analilys. 41: 1508-1522.

Moreno, D.A., Pérez-Balibrea, S., Ferreres, F., Gil-Izquierdo, Á. y García-Viguera, C. 2010. Acylated anthocyanins in broccoli sprouts. Food Chemistry. 123: 358-363.

Olle, M. y Viršile, A. 2013. The effects of light-emitting diode lighting on greenhouse plant growth and quality. Agricultural and Food Science. 22: 223-234.

Paniagua-Pardo, G., Hernández-Aguilar, C., Rico-Martínez, F., Domínguez-Pacheco, F.A., Martínez-Ortiz, E. y MartínezGonzález, C.L. 2015. Efecto de la luz led de alta intensidad sobre la germinación y el crecimiento de plántulas de brócoli (Brassica oleracea L.). Polibotánica. 40: 199-212.

Pérez-Balibrea, S., Moreno, D.A. y García-Viguera, C. 2008. Influence of light on health-promoting phytochemicals of broccoli sprouts. Journal of the Science of Food and Agriculture. 88: 904-910.

Pérez-Nájera, V.C., Lugo, E., Gutiérrez-Lomelí, M. y Del Toro, L. 2013. Extracción de compuestos fenólicos de la cáscara de lima (Citrus limetta risso) y determinación de su actividad antioxidante. Biotecnia. 15: 18-22.

Perez-Perez, L.M., García-Borbón, L., González-Vega, R.I., José Carlos Rodríguez-Figueroa, J.C., Rosas-Burgos, E.C., HuertaOcampo, J.A., Ruiz-Cruz, S., Wong-Corral, F.J., Borboa-Flores, J., Rueda-Puente, E.O. y Del-Toro-Sánchez, C.L. 2018. Liberación de compuestos fenólicos ligados en el garbanzo (Cicer arietinum L.) utilizando microbiota humana intestinal. Biotecnia. 20(3), 146-154.

Prior, R.L., Wu, X. y Schaich, K. 2005. Standardized methods for the determination of antioxidant capacity and phenolics in foods and dietary supplements. Journals Agricultural and Food Chemestry. 53: 4290-4302.

Qian, H., Liu, T., Deng, M., Miao, H., Cai, C., Shen, W. y Wang, Q. 2016. Effects of light quality on main health-promoting compounds and antioxidant capacity of Chinese kale sprouts. Food Chemestry. 196: 1232-1238.
Ramírez-Rojo, M. I., Vargas-Sánchez, R. D., del Mar TorresMartínez, B., Torrescano-Urrutia, G. R., y Sánchez-Escalante, A. (2018). Extractos de hojas de plantas para conservar la calidad de la carne y los productos cárnicos frescos. Revisión. Biotecnia. 20(3), 155-164.

Re, R., Pellegrini, N., Proteggente, A., Pannala, A., Yang, M. y RiceEvans, C. 1999. Antioxidant activity applying an improved ABTS radical cation decolorization assay. Free Radical Biology \& Medicine. 26: 1231-1237.

Rodríguez-Hernández, M.D.C., Moreno, D.A., Carvajal, M. y Martínez-Ballesta, M.D.C. 2014. Genotype Influences Sulfur Metabolism in Broccoli (Brassica oleracea L.) Under Elevated $\mathrm{CO} 2$ and $\mathrm{NaCl}$ Stress. Plant and Cell Physiology. 55: 20472059.

Sánchez-García, Y., Rondón-Arias, L., Hermosilla-Espinosa, R. y Almeida- Saavedra, M. 2010. Tamizaje fitoquímico de los extractos alcohólico, etéreo y acuoso de las hojas, tallos y flores de la Helychrysum bracteatum. Química Viva. 9: 40-45.

Sikin, A.M., Zoellner, C. y Rizvi, S.S. 2013. Current intervention strategies for the microbial safety of sprouts. Journal Food Protection. 76: 2099-2123.

Solanilla, D.J.F., Lombo, O., Perae, M.E. Y Méndez, A.J.J. 2011. Valoración del potencial antioxidante de Mollinedia racemosa (romadizo). Revista Cubana de Plantas Medicinales. 16: 151-163.Tian, X. y Schiach, K.M. 2013. Effects of molecular structure on kinetics and dynamics of the trolox equivalent antioxidant capacity assay with ABTS ${ }^{\text {. Journals of }}$ Agricultural and Food Chemistry. 61: 5511-5519.

Tong-Jen F., Reineke, K.F., Chirtel, S. y VanPelt, O.M. 2008. Factors influencing the growth of Salmonella during sprouting of naturally contaminated alfalfa seeds. Journal of food protection. 71: 888-896.

Vale, A.P., Cidade, H., Pinto, M. y Oliveira, M.B. 2014. Effect of sprouting and light cycle on antioxidant activity of Brassica oleracea varieties. Food Chemestry. 165: 379-87.

Verkerk, R., Schreiner, M., Krumbein, A., Ciska, E., Holst, B., Rowland, I., De Schrijver, R., Hansen, M., Gerhauser, C., Mithen, R. y Dekker, M. 2009. Glucosinolates in Brassica vegetables: the influence of the food supply chain on intake, bioavailability and human health. Molecular Nutrition Food Research. 53: S219-S265. 